\title{
THE COLD PRESSOR TEST - A MEASUREMENT OF THE CARDIAC AUTONOMIC RESPONSES IN INDIVIDUALS WITH ANXIETY, DEPRESSION AND STRESS SYMPTOMS
}

\author{
E. Nikolov* \\ Faculty of Medicine, Medical University - Sofia, Sofia, Bulgaria
}

\begin{abstract}
The cold pressor test examines the cardio-vascular reactivity during certain physiological states - rest, stress and recovery. Currently it is a widespread tool for laboratory induced stress in an experimental setting amongst various fields of science. PURPOSE: To systemize the fields of application of the cold pressor test in an attempt to outline the possibilities to analyze cardio-vascular reactivity in connection to symptoms of depression, anxiety and stress. RESULTS: Unlike similar experiments (e.g. mental arithmetic test), which actively involve the behavior and motivation of the participants, the cold pressor test is a physical stressor that passively involves the participant. Cardiovascular response (BP, heart rate, aortic pulse wave velocity) to cold pressor test is measured with applanation tonometry - a novel, simple, easy-to-use method. In CONCLUSION, the combination of the cold pressor test with speech task and mental arithmetic test would contribute to the adequate tracking and comparison of vascular and myocardial reactivity during the assessment of psychological symptoms (depression, anxiety, and stress). All of this would facilitate the involvement of the individuals with clinical or sub-clinical symptoms of depression and anxiety in experimental studies and would further help in a more precise assessment of the cardiovascular risk amongst them.
\end{abstract}

Key words: Cardiovascular reactivity, applanation tonometry, cardiovascular risk, stressor

\section{INTRODUCTION}

Stress is considered as an adaptive reaction to variety of different harmful stimuli or situations. The stress reaction is based on a complicated sudden change in the organism's physiological and psychological functioning (1). On a physiological level, the organism distributes its resources in order to quickly execute preventing actions at the cost of a longer regenerative period. Acute stress incorporates an endocrinal response (2) and induces a sympathetic response (3), thus leading to a change in somatic motor behaviour and psychological adjustments.

The cold pressor test (CPT) is a procedure in which the wrist of the hand (typically the dominant one) is put in ice-cold water for a period of 2-3 minutes. It was described for the first time by Hines and Brown (4). They

*Correspondence to: Emil Nikolov, Faculty of Medicine, Medical University - Sofia, Sofia 1000, Bulgaria, E-mail address: e.vnikolov@outlook.com, Tel: +359882470664 introduced a procedure, beginning with a resting period, in which time a series of measurements of the blood pressure are taken. This is followed by the main cold pressor test and a final recovery period. These latter two are accompanied by blood pressure measurements as well. This method of testing the responsiveness during rest, stress and recovery is still applied as a basic procedure for stress investigation. Although the initial intent of the authors was to create a systemized way of experimentally raising the blood pressure in hypertension studies, the CPT has been transformed into a widespread tool for laboratory induced stress under experimental settings amongst various fields of science.

The typical cardiovascular cold pressor effect consists of systolic and/or diastolic BP raise by $10-20 \mathrm{mmHg}$ combined with increased heart rate (5). The pressor response is characterized by an increased cardiac output in the initial period (0-30 s) (6), followed by increased sympathetic muscle tone (30-120 s). Although 
there is a fair fluctuation of the results for the heart rate, most authors describe a similar cardiovascular response $(7,8)$. The interpretation of the $\mathrm{CP}$ effect is widely researched but most studies reach the conclusion that it involves primary neurogenic reflexes with triggering activity of the peripheral autonomic structures, leading to activation of the spinal cord, thalamus, sensory cortex and autonomic nervous system (9).

Thermo and nociceptors, located throughout the skin, are the entry gate of the stimulation caused by CPT exposure. Entering the dorsal roots of the spinal cord, the nerve fibers cross contralateral to form the spinolateral tract which travels to the thalamus and sends collaterals to the reticular formation. As the collaterals reach the medulla a reflexive sympathetic discharge towards the heart and the vessels is sent as a result from rostral ventrolateral medullary pressor area stimulation $(10,11)$. The cortical and subcortical structures may also be affected by the CPT stimulation and further modulate the neuroendocrine response resulting in a complex multi-level physiological and psychological stress response $(9,12,3)$.

As mentioned above, the most notable changes in cardiovascular parameters in response to the CPT consist of the rise in blood pressure through peripheral vasoconstriction and to a lesser extent cardiac output during which an increase in both vascular alpha-adrenergic and cardiac beta-adrenergic drive is observed (9, 6). Nevertheless, CPT provokes an increase in multiple markers of sympathetic nervous system activity as skin conductance level (13), plasma catecholamines $(14,15)$, muscle sympathetic nerve activity (6) salivary alphaamylase (sAA) (16). Additionally, the HPA axis is reported to be activated, as McRae et al. (17) found elevated plasma ACTH concentration after CPT exposition. Cortisol concentrations in saliva samples are shown to be elevated about 15 minutes after the CPT (18; 19). Participants experience the CPT as painful and demonstrate increased levels of perceived stress and arousal during and immediately after the experiment, which demonstrates changes on the subjective level as well $(18,20)$.

\section{THE COLD PRESSOR TEST IN PSYCHOPHYSIOLOGICAL RESEARCH}

Originally, the CPT was intended as an experiment of increasing the blood pressure under laboratory settings in studies on etiology of hypertension. However, in the recent decades it has been adapted to a wide range of psychophysiological studies. Despite the lack of consensus on the results (21), hyperreactivity in blood pressure responses to CPT (higher than $20 \mathrm{mmHg}$ ) has been reported as an early predictor of the development and severity of essential hypertension (22).

The sympathetic integrity and the normal cardiovascular functioning among both healthy and clinical populations have also been investigated with the help of the CPT in studies of the severity of autonomic dysfunction in diabetes mellitus and spinal cord injury (23). More studies introduce the CPT as a pain inducing method which helps evaluate the analgesic effect of pharmacological and psychological treatments (24).

Both cortisol and catecholamines are of major interest for psychophysiological stress research. As the CPT causes elevated levels of these hormones, it has been employed as a convenient laboratory stressor. CPT has been proven to alter a various amount of psychophysiological phenomena like autonomic startle responses (25) as well as the cardiac modulation of the startle response (26). Behavioral and electrophysiological indices demonstrate how CPT impairs the top-down control of attention (27). In addition, stress effects on memory processes have also been studied using CPT. It has been proved to impair retrieval processes while enhancing consolidation of diverse classes of stimuli (19).

\section{ADVANTAGES OF THE COLD PRESSOR TEST}

The CPT is a fairly simple experiment yet widely used across diverse fields of study. The variety of different outcome variables ranging from plasma and salivary concentrations of (neuro-hormones $(15,16)$ over electrophysiological parameters $(6,13)$ to subjective reports $(18,20)$ demonstrate the test's wide range of advantages. Interindividual differences that influence the CPT reactions are also widely investigated in different studies $(22,28)$.

The information and research on CPT is so comprehensive that the researchers could predict the outcomes and take into considerations all the different factors. The 
little time needed for preparation and application makes it an economic laboratory stress inducer and allows for an accurate timing of the experiment. Moreover, CPT is a passive task which does not cause any cognitive load on the subjects, unlike the active tasks (e.g. mental arithmetic, public speaking task) and thus helps to reduce conflicts with other experimental measures.

\section{BLOOD PRESSURE REACTIVITY AND HYPERTENSION - NORMOREACTORS AND HYPERREACTORS}

Vascular reactivity to the CPT is measured by the difference between peak and basal blood pressure. Subjects are classified according to their response to the cold pressor test. Individuals with an increase in blood pressure of at least $25 \mathrm{mmHg}$ systolic or $20 \mathrm{mmHg}$ diastolic are classified as hyper-reactors whereas the normoreactors are subjects with an increase less than that $(29,30)$.

Menkes and coauthors found a significant independent association between blood pressure reactivity to the CPT and incidence of subsequent hypertension (30). 2 other prospective studies achieved similar results. Subjects classified as hyper-reactors to the CPT at either of the two time periods were found to be at a 3,7-fold risk of hypertension, according to Wood et al (29) who followed $47 \%$ of a study cohort of 300 school students for 45 years. Barnett and associates found a $10 \%$ (4/40) incidence of hypertension among hyper-reactors at the first time of the experiment, but no hypertension among normoreactors.

Other prospective studies have not found a significant association between cardiovascular cold pressor reactivity and subsequent hypertension. For example, an 18-year followup study of a cohort of aviators, a group that is healthy and physically fit, with expected lower overall heart rate and blood pressure. A possible explanation as to why it didn't find a significant correlation was that by the end of the 18-year follow up the mean age of the group was 41 years, an age considered to be too early for the development of hypertension. The prevalence of hypertension dramatically increases with age. Thus, an assumption is made that the assessment of the end point should not be made until the targeted population reaches a mean age of $45-50$ years.
That is when a precise and comprehensive conclusion can be made.

It is important to note that the CPT differs from experiments which require active coping by the hemodynamic mechanisms it provokes. CPT is associated with elevated levels of norepinephrine and higher blood pressure response compared to the mental arithmetic test which elicits higher levels of plasma epinephrine associated with elevated heart rate (31). Norepinephrine levels are found to be more strongly correlated with blood pressure levels than with heart rates. Tasks like the mental arithmetic test, shock avoidance, or other situational demands that require "active coping" and trigger affective behaviors are speculated to be predictive for hypertension. Ditto, 1986 suggests that subjects with a family history of hypertension indeed tend to express higher cardiovascular responsiveness.

However, most studies fail to clarify why episodic cardiovascular hyperresponsiveness is related to risk for hypertension. It might be, in fact, one of the mechanisms underlying essential hypertension, should it represent a central defect in the autonomic nervous system, or indicate changes in arterial compliance.

A recent study (32) suggested that the CPT provoked a greater sodium efflux in leukocytes of subjects with a family history of hypertension compared to those with a negative history. In addition to this intriguing observation, other researchers have concluded that there are sodium-lithium transport abnormalities in blood cell membranes in patients with essential hypertension. This leads to the proposal (40-41) that pressor hyperactivity is a manifestation of a widespread basic membrane transport disorder disrupting cellular cation homeostasis.

The Menkes and associates study (30) suggests that elevated blood pressure reactivity, especially systolic BP, could prove as an independent predictor of early hypertension. That is how CPT could potentially be helpful in identifying a group of individuals with this physiological abnormality and warrant preventive actions among these individuals.

\section{APPLANATION TONOMETRY}

Pulse Wave Velocity (PWV) is a measure of arterial elasticity, or the rate at which pressure waves move down the vessels. This is why 
PWV is a reliable predictor of the development of cardiovascular diseases. Applanation tonometry is a novel method of measuring PWV which is simple, easy-to-use and photoplethysmography-based Multi Photodiode Array (MPA) which could prove to provide measurements that are just as accurate as the ones made with more complicated and harder-to-use systems.

The cardiovascular responses (BP, heart rate, aortic pulse wave velocity) to CPT can be recorded by using the applanation tonometry. The pulse wave velocity (PWV) measurements are performed using a SphygmoCor apparatus (SphygmoCor system, AtCor Medical, Sydney, Australia) after a 10-min rest (supine position) (33). To minimize the effect of the circadian cycle, measurements are taken at approximately the same time in the morning on an empty stomach after instructions to refrain from any beverages of caffeine, ethanol and smoking at least 12 hours prior to the estimates (34).

PWV is registered between the carotid and femoral artery in the supine position. The SphygmoCor probe over the carotid and femoral artery is used for non-invasive pulse measurements (35). The values of the distance from the carotid to femoral artery, measured directly between artery location and the suprasternal notch are entered into the SphygmoCor software database. PWV was automatically calculated using AtCor software

\section{DEPRESSION, ANXIETY, STRESS AND CARDIOVASCULAR DISEASE}

The mechanisms connecting depression, anxiety, and cardiovascular disease (CVD) are complex and are yet to be fully understood. A possible explanation linking psychological factors (depression and anxiety) to CVD and cardiovascular mortality is cardiovascular reactivity. A meta-analysis of 11 studies conducted from 1887 to 2001 supports the hypothesis that exaggerated responses to stress were associated with depression (36). They find a small to moderate positive correlation between cardiovascular reactivity and depressive symptoms. On the other hand, these results didn't reach the criteria for statistical significance. Recent studies that support the same hypothesis report that there may be a weak, positive relationship between depression and anxiety symptoms and cardiovascular reactivity. Pointer et al. (37) studied a population of 50 healthy adults and found that state anxiety was positively associated with blood pressure (BP) responses to cold pressor and anger recall. In another study of 60 healthy young women who completed a speech task, depressive symptoms were positively associated with $\mathrm{BP}$, heart rate (HR), and cardiac output (CO) responses (38).

Other studies report no difference in cardiovascular reactivity between depressed and non-depressed subjects. In a study of the cardiovascular reactions to the Tier Social Stress Test Taylor et al. examined 59 older depressed patients and 20 non-depressed subjects matched for age and cardiovascular risk. The results pointed out that there are no significant differences in blood pressure reactivity between depressed and nondepressed individuals (39). The small sample size and lack of technical equipment though, might be the reason for such results. Studies of similar size, however, have detected significant positive correlations.

On the other hand, many studies have found that anxiety and depressive symptoms are negatively associated with cardiovascular reactivity. The largest study to date (carol et al.), which involved 1608 adults with depressive symptoms, concluded that depression scores were negatively associated with systolic blood pressure (SBP) and HR reactions to the Paced Auditory Serial Arithmetic Test (PASAT) (40). Trait anxiety was reported to be negatively correlated to cardiovascular reactivity in a study amongst 832 healthy participants, which compared those with higher levels of trait anxiety to participants with low levels of anxiety (41). The hypothesis that anxiety and depression is related to a blunted physiological reactivity is supported by a number of studies including a meta-analysis by Chida \& Hamer of studies from 1950 to 2008 (42). All things said, it is clear that more recent studies conducted among larger populations tend to claim the presence of negative relationships of symptoms of anxiety and depression with cardiovascular reactivity.

Another detail to take into consideration is the fact that most studies examined cardiovascular responses only to a single task. To date, there are two studies that put healthy participants through both an active and passive coping task (43). Pointer et al. investigated BP responses to 
both active and passive coping tasks (anger recall and cold pressor) in 50 adults and reported a positive correlation between state anxiety and BP responses to both active and passive coping tasks (anger recall and cold pressor test). However, another study conducted by Schwerdtfeger \& Rosenkaimer found negative association between depressive symptoms and $\mathrm{BP}$ responses to an active coping task (public speaking), yet no association was found with BP responses to a passive coping task (cold pressor test) (44). A possible explanation of the contradicting results is the small sample size as well as the exclusion of participants in the process of the examination.

Table 1. Studies of the cold pressor test and cardiac autonomic responses

\begin{tabular}{|c|c|c|c|}
\hline Subject of Study & Authors & Correlations & $\begin{array}{l}\text { Number of } \\
\text { participants }\end{array}$ \\
\hline \multirow{5}{*}{$\begin{array}{l}\text { Cardio-vascular } \\
\text { responsiveness and } \\
\text { hypertension }\end{array}$} & Lambert, E. A., and Schlaich, M. P., 2004 & $\uparrow$ & 32 \\
\hline & $\begin{array}{l}\text { Flaa, A., Eide, I. K., Kjeldsen, S. E., \& } \\
\text { Rostrup, M., } 2008\end{array}$ & $\uparrow$ & 99 \\
\hline & $\begin{array}{l}\text { Previnaire, J. G., Soler, J. M., Leclercq, V., } \\
\text { and Denys, P., } 2012\end{array}$ & $\leftrightarrow$ & 45 \\
\hline & $\begin{array}{l}\text { Wood, D. L., Sheps, S. G., Elveback, L. R., } \\
\text { and Schirger, A., } 1984\end{array}$ & $\uparrow$ & 142 \\
\hline & $\begin{array}{l}\text { Menkes, M. S., Matthews, K. A., Krantz, D. } \\
\text { S., Lundberg, U., Mead, L. A., Qaqish, B., ... } \\
\text { and Pearson, T. A., } 1989\end{array}$ & $\leftrightarrow$ & 910 \\
\hline \multirow{6}{*}{$\begin{array}{l}\text { Cardio-vascular } \\
\text { responsiveness, } \\
\text { depression and anxiety }\end{array}$} & Kurtz, M. M., \& Mueser, K. T., 2008 & $\uparrow$ & 1599 \\
\hline & $\begin{array}{l}\text { Pointer, M. A., Yancey, S., Abou-Chacra, } \\
\text { R., Petrusi, P., Waters, S. J., and } \\
\text { McClelland, M. K., } 2012\end{array}$ & $\uparrow$ & 179 \\
\hline & $\begin{array}{l}\text { Salomon, K., Clift, A., Karlsdóttir, M., and } \\
\text { Rottenberg, J., } 2009\end{array}$ & $\uparrow$ & 50 \\
\hline & $\begin{array}{l}\text { Young, E. A., Nesse, R. M., Weder, A., and } \\
\text { Julius, S., } 1998\end{array}$ & $\downarrow$ & 832 \\
\hline & $\begin{array}{l}\text { Yuenyongchaiwat, K., Baker, I. S., and } \\
\text { Sheffield, D., } 2017\end{array}$ & $\downarrow$ & 104 \\
\hline & $\begin{array}{l}\text { Buckman, J. F., Vaschillo, B., Vaschillo, E. } \\
\text { G., Epstein, E. E., Nguyen-Louie, T. T., } \\
\text { Lesnewich, L. M., ,.. and Bates, M. E., } 2019\end{array}$ & $\uparrow$ & 92 \\
\hline
\end{tabular}

Recent studies point out a possibility that cardiovascular reactivity may be related to negative health outcomes and behaviors (45) Motivation is thought to be a possible mechanism. Specifically, the lower cardiovascular reactivity may be a physiological marker of motivational dysregulation, which is a basic symptom in patients with anxiety and depression (46). (Table 1) Patients with lower cardiovascular reactivity are reported to display increased scores of anxiety and depression. 8 Moreover, Salomon et al. found that participants with major depression rated speech and mirrortracing tasks as more demanding, threatening, and stressful than participants without major depression These subjective ratings, of course, alone couldn't explain the relationship between the lower cardiovascular reactivity and depression. As in the above-mentioned studies, these two subjected participants only to an active coping task as well.

\section{CONCLUSIONS}

The mechanisms underlying the cardiovascular response to active and passive coping tasks differ from each other. Active coping tasks involve beta-adrenergic nerve fibers which increase BP via central mechanism through increases in catecholamines, HR, systolic blood pressure, stroke volume, and cardiac output. Passive coping tasks, on the other hand, are thought to cause changes in diastolic BP and total peripheral resistance by vasoconstriction, mediated by alpha-adrenergic receptor stimulation. That being said, active 
coping tasks express myocardial reactivity patterns (by beta-adrenergic stimulation) whereas passive coping tasks are connected to vascular reactivity patterns.

In conclusion, the combination of the cold pressor test with speech task and mental arithmetic test would contribute to the adequate tracking and comparison of vascular and myocardial reactivity during the assessment of psychological symptoms (depression, anxiety, and stress). The method of applanation tonometry would make such a research more accurate, as well as simpler in turn of realization and experimental setup. All of this would facilitate the involvement of the individuals with clinical or sub-clinical symptoms of depression and anxiety in experimental studies and would further help in a more precise assessment of the cardiovascular risk amongst them.

\section{REFERENCES}

1. Del Giudice, M., Ellis, B.J. and Shirtcliff, E.A. The adaptive calibration model of stress responsivity. Neurosci. Biobehav. Rev., $\quad 35.7$ : 1562-1592, 2011. https://doi.org/10.1016/j.neubiorev.2010.11 .007

2. de Kloet, E. R. Hormones, brain and stress. Endocr Regul, 37(2), 51-68, 2003.

3. Ulrich-Lai, Y. M., and Herman, J. P. Neural regulation of endocrine and autonomic stress responses. Nat. Rev. Neurosci., 10(6), 397-409, https://doi.org/10.1038/nrn2647

4. Hines, E. A., and Brown, G. E. The hereditary factor in the reaction of blood pressure to a standard stimulus. In Proc. Staff Meeting Mayo Clinic (Vol. 7, p. 332), 1932.

5. Simoes GM, Campagnaro BP, Tonini CL, Meyrelles SS, Kuniyoshi FH, Vasquez EC. Hemodynamic reactivity to laboratory stressors in healthy subjects: influence of gender and family history of cardiovascular diseases. Int J Med Sci,. 10(7):848-856, 2013. doi:10.7150/ijms.5967

6. Yamamoto, K., Iwase, S., and Mano, T. Responses of muscle sympathetic nerve activity and cardiac output to the cold pressor test. The Japanese journal of physiology, 42(2), 239-252, 1992. https://doi.org/10.2170/jjphysiol.42.239

7. Willemsen, G., Ring, C., Carroll, D., Evans, P., Clow, A., and Hucklebridge, F. Secretory immunoglobulin A and cardiovascular reactions to mental arithmetic and cold pressor. Psychophysiology, 35(3), 252-259, 1998. https://doi.org/10.1111/1469-8986.3530252

8. Ring, C., Harrison, L. K., Winzer, A., Carroll, D., Drayson, M., and Kendall, M. Secretory immunoglobulin A and cardiovascular reactions to mental arithmetic, cold pressor, and exercise: Effects of alpha- adrenergic blockade. Psychophysiology, 37(5), 634-643, 2000. https://doi.org/10.1111/1469-8986.3750634

9. Lovallo, W. The cold pressor test and autonomic function: a review and integration. Psychophysiology, 12(3), 268282, 1975. https://doi.org/10.1111/j.14698986.1975.tb01289.x

10.Kawano, H., Tanimoto, M., Yamamoto, K., Sanada, K., Gando, Y., Tabata, I., ... and Miyachi, M. Resistance training in men is associated with increased arterial stiffness and blood pressure but does not adversely affect endothelial function as measured by arterial reactivity to the cold pressor test. Exp. Physiol., 93(2), 296-302, 2008. https://doi.org/10.1113/expphysiol.2007.03 9867

11.Velasco, M., Gómez, J., Blanco, M., and Rodriguez, I. The cold pressor test: pharmacological and therapeutic aspects. Am J Ther, 4(1), 34-381, 1997.

12.McEwen, B. S. Physiology and neurobiology of stress and adaptation: central role of the brain. Physiol. Rev., 87(3), 873-904, 2007.

13.Buchanan, T. W., Tranel, D., and Adolphs, R. Impaired memory retrieval correlates with individual differences in cortisol response but not autonomic response. Learn. Mem., 13(3), 382-387, 2006. http://www.learnmem.org/cgi/doi/10.1101/l m.206306

14.Goldstein, B., Woolf, P. D., Deking, D., Delong, D. J., Cox, C., and Kempski, M. H. Heart rate power spectrum and plasma catecholamine levels after postural change and cold pressor test. Pediatr. Res., 36(3), 358-363,

1994. https://doi.org/10.1203/00006450199409000-00016

15.Pascualy, M., Petrie, E. C., Brodkin, K., Peskind, E. R., Wilkinson, C. W., and Raskind, M. A. Hypothalamic pituitary adrenocortical and sympathetic nervous system responses to the cold pressor test in Alzheimer's disease. Biol. psychiatry, 48(3), 247-254, 2000. 
https://doi.org/10.1016/S0006-

3223(00)00879-9

16.Smeets, T., Otgaar, H., Candel, I., and Wolf, O. T. True or false? Memory is differentially affected by stress-induced cortisol elevations and sympathetic activity at consolidation and retrieval. Psychoneuroendocrinology, 33(10), 13781386, 2008. https://doi.org/10.1016/j.psyneuen.2008.07. 009

17.McRae, A. L., Saladin, M. E., Brady, K. T., Upadhyaya, H., Back, S. E., and Timmerman, M. A. Stress reactivity: biological and subjective responses to the cold pressor and Trier Social stressors. Hum Psychopharmacol, 21(6), 377-385, 2006. https://doi.org/10.1002/hup.778

18.al'Absi, M., Petersen, K. L., and Wittmers, L. E. Adrenocortical and hemodynamic predictors of pain perception in men and women. Pain, 96(1-2), 197-204, 2002. https://doi.org/10.1016/S03043959(01)00447-X

19.Felmingham, K. L., Tran, T. P., Fong, W. C., and Bryant, R. A. Sex differences in emotional memory consolidation: the effect of stress-induced salivary alpha-amylase and cortisol. Biol Psychol, 89(3), 539-544, 2012.

https://doi.org/10.1016/j.biopsycho.2011.12 .006

20.Zoladz, P. R., Peters, D. M., Kalchik, A. E., Hoffman, M. M., Aufdenkampe, R. L., Woelke, S. A., ... and Talbot, J. N. Brief, pre-learning stress reduces false memory production and enhances true memory selectively in females. Physiol. Behav., 128, 270-276, 2014.

21.Lambert, E. A., and Schlaich, M. P. Reduced sympathoneural responses to the cold pressor test in individuals with essential hypertension and in those genetically predisposed to hypertension: no support for the "pressor reactor" hypothesis of hypertension development. Am. J. Hypertens., 17(10), 863-868, 2004. https://doi.org/10.1016/j.amjhyper.2004.05. 008

22.Flaa, A., Eide, I. K., Kjeldsen, S. E., \& Rostrup, M. Sympathoadrenal stress reactivity is a predictor of future blood pressure: an 18-year follow-up study. Hypertension, 52(2), 336-341, 2008. https://doi.org/10.1161/HYPERTENSION AHA.108.111625
23.Previnaire, J. G., Soler, J. M., Leclercq, V., and Denys, P. Severity of autonomic dysfunction in patients with complete spinal cord injury. Clin. Auton. Res., 22(1), 9-15, 2012.

24.Edwards, R. R., Campbell, C. M., and Fillingim, R. B. Catastrophizing and experimental pain sensitivity: only in vivo reports of catastrophic cognitions correlate with pain responses. J Pain, 6(5), 338-339, 2005.

https://doi.org/10.1016/j.jpain.2005.02.013

25.Deuter, C. E., Kuehl, L. K., Blumenthal, T. D., Schulz, A., Oitzl, M. S., and Schachinger, H. Effects of cold pressor stress on the human startle response. PloS one, 7(11), e49866, 2012. https://doi.org/10.1371/journal.pone.00498 66

26.Schulz, A., Plein, D. E., Richter, S., Blumenthal, T. D., and Schächinger, $\mathrm{H}$. Cold pressor stress affects cardiac attenuation of startle. Int J Psychophysiol, 79(3), 385-391, 2011. https://doi.org/10.1016/j.ijpsycho.2010.12.0 08

27. Sänger, J., Bechtold, L., Schoofs, D., Blaszkewicz, M., and Wascher, E. The influence of acute stress on attention mechanisms and its electrophysiological correlates. Front. Behav. Neurosci., 8, 353, 2014.

https://doi.org/10.3389/fnbeh.2014.00353

28.Wu, T., Snieder, H., and de Geus, E.Genetic influences on cardiovascular stress reactivity. Neurosci. Biobehav. Rev., 35(1), 58-68, 2010. https://doi.org/10.1016/j.neubiorev.2009.12 .001

29.Wood, D. L., Sheps, S. G., Elveback, L. R., and Schirger, A. Cold pressor test as a predictor of hypertension. Hypertension 6, 301-306, $1984 . \quad$ doi: 10.1161/01.hyp.6.3.301

30.Menkes, M. S., Matthews, K. A., Krantz, D. S., Lundberg, U., Mead, L. A., Qaqish, B., $\ldots$ and Pearson, T. A. Cardiovascular reactivity to the cold pressor test as a predictor of hypertension. Hypertension, 14(5), 524-530, 1989.

31.Andrén, L., Hansson, L., \& Section, H. Circulatory effects of stress in essential hypertension. Acta Medica Scandinavica, 209(S646), 1981. https://doi.org/10.1111/j.09546820.1981.tb02623.x 
32.Umana E., Ahmed W., Fraley M.A. and Alpert M.A. Comparison of oscillometric and intraarterial systolic and diastolic blood pressures in lean, overweight, and obese patients. Angiology. 57(1):41-52006.

33. Townsend, R. R. Arterial stiffness and chronic kidney disease: lessons from the Chronic Renal Insufficiency Cohort study. Curr. Opin. Nephrol. Hypertens., 24(1), 47, 2015.

https://dx.doi.org/10.1097\%2FMNH.00000 00000000086

34.Papaioannou, T. G., Lekakis, J. P., Karatzis, E. N., Papamichael, C. M., Stamatelopoulos, K. S., Protogerou, A. D., ... and Stefanadis, C. Transmission of calibration errors (input) by generalized transfer functions to the aortic pressures (output) at different hemodynamic states. Int. J. Cardiol., 110(1), 46-52, 2006. https://doi.org/10.1016/j.ijcard.2005.07.014

35.Qureshi, G., Brown, R., Salciccioli, L., Qureshi, M., Rizvi, S., Farhan, S., and Lazar, J. Relationship between aortic atherosclerosis and non-invasive measures of arterial stiffness. Atherosclerosis, 195(2), e190-e194,

2007. https://doi.org/10.1016/j.atherosclerosis.200 7.06.021

36.Kurtz, M. M., \& Mueser, K. T. Metaanalysis of controlled research on social skills training for schizophrenia. $J$ Consult Clin Psychol, 76(3), 491, 2008. https://psycnet.apa.org/doi/10.1037/0022006X.76.3.491

37.Pointer, M. A., Yancey, S., Abou-Chacra, R., Petrusi, P., Waters, S. J., and McClelland, M. K. State anxiety is associated with cardiovascular reactivity in young, healthy African Americans. Int. J. Hypertens.,

2012. https://doi.org/10.1155/2012/268013

38.Salomon, K., Clift, A., Karlsdóttir, M., and Rottenberg, J. Major depressive disorder is associated with attenuated cardiovascular reactivity and impaired recovery among those free of cardiovascular disease. Health psychol, 28(2), 157, 2009.

39.Du, X., and Pang, T. Y Is dysregulation of the HPA-axis a core pathophysiology mediating co-morbid depression in neurodegenerative diseases? Front.
NIKOLOVE.

Psychiatry, 6, 32, 2015. https://doi.org/10.3389/fpsyt.2015.00032

40. Yuenyongchaiwat, K. Do haemodynamic responses to mental stress tests predict future blood pressure one year later? Prospective studies in the United Kingdom and $\quad 2013$. http://hdl.handle.net/10545/301912

41. Young, E. A., Nesse, R. M., Weder, A., and Julius, S. Anxiety and cardiovascular reactivity in the Tecumseh population. $J$. Hypertens., 16(12), 1727-1733, 1998.

42. Yuenyongchaiwat, K., Baker, I. S., and Sheffield, D. Symptoms of anxiety and depression are related to cardiovascular responses to active, but not passive, coping tasks. Rev. Bras. Psiquiatr, 39(2), 110-117, 2017. https://doi.org/10.1590/1516-44462016-1935

43.Salovey, P., Stroud, L. R., Woolery, A., and Epel, E. S. (). Perceived emotional intelligence, stress reactivity, and symptom reports: Further explorations using the trait meta-mood scale. Psychol Health, 17(5), 611-627, 2002. https://doi.org/10.1080/0887044029002581 2

44.Schwerdtfeger A, Rosenkaimer AK. Depressive symptoms and attenuated physiological reactivity to laboratory stressors. Biol Psychol.;87:430-8, 2011.

45.Buckman, J. F., Vaschillo, B., Vaschillo, E. G., Epstein, E. E., Nguyen-Louie, T. T., Lesnewich, L. M., ... and Bates, M. E. Improvement in women's cardiovascular functioning during cognitive-behavioral therapy for alcohol use disorder. Psychol Addict Behav, 33(8), 659, 2019. https://psycnet.apa.org/doi/10.1037/adb000 0524

46.Mennin, D. S., Heimberg, R. G., Turk, C. L., and Fresco, D. M. Preliminary evidence for an emotion dysregulation model of generalized anxiety disorder. Behav Res Ther, 43(10), 1281-1310, 2005. https://doi.org/10.1016/j.brat.2004.08.008

47.Kline, K. A. Cardiovascular, affective, and behavioral responses to masculinitychallenging stressors: Active versus passive coping. Psychol Men Masc, 19(1), 35, 2018.

https://psycnet.apa.org/doi/10.1037/men000 0070 